\title{
Indication of pulsation in young Brown Dwarfs
}

\author{
M. Marconi, ${ }^{1}$ V. Ripepi, ${ }^{1}$ M. Oliviero, ${ }^{1}$ L. Errico, ${ }^{1}$ M. Magrí, ${ }^{1}$ A. Vittone, ${ }^{1}$ F. Palla ${ }^{2}$ \\ S. Bernabei ${ }^{3,4}$ \\ ${ }^{1}$ INAF-OACapodimonte, Via Moiariello 16, 80131, Napoli, Italy \\ 2 INAF-OAAarcetri, Largo E. Fermi, 5, I-50125, Firenze, Italy \\ 3 INAF-OABologna, Via Ranzani 1,40127 Bologna, Italy \\ ${ }^{4}$ Univ. de La Laguna, Avda. Astrofisico F. Sánchez sn, 30071 La Laguna, Spain
}

\begin{abstract}
Preliminary results of a project devoted to the detection of pulsation in young brown dwarfs are presented. In particular, we show the light curve and frequency analysis for the first candidate, CFHT BD3 in the Taurus-Auriga association. Future plans are briefly discussed.
\end{abstract}

\section{Introduction}

New classes of pulsators have been recently discovered or predicted and could be confirmed and classified with the advent of space missions like COROT. As an example, Palla \& Baraffe (2005, hereinafter PB05) have theoretically predicted the existence of pulsating young Brown Dwarfs (BDs) during the central deuterium burning phase, with typical time scales ranging from $\sim 1 \mathrm{~h}$ for $0.02 \mathrm{M}_{\odot}$ to $\sim 5 \mathrm{~h}$ for $0.1 \mathrm{M}_{\odot}$. Furthermore, PB05 identify possible candidates for pulsational variability among known BDs in nearby star forming regions whose location in the HR diagram falls within or close to the instability strip. To test this suggestion, we have started a program dedicated to the observation of potentially interesting candidates. In particular, during winter 2005 we used the Asiago $(1.8 \mathrm{~m})$ and Loiano $(1.5 \mathrm{~m})$ telescopes to observe CFHT BD2 and CFHT BD3, two BDs in the Tau-Aur association that fall into the PB05 instability strip (see Fig. 1). Unfortunately, we were only able to observe for one night at each site (separated by 10 days), for a total of $7.8 \mathrm{~h}$ and $4.2 \mathrm{~h}$ of observing time in Asiago and Loiano, respectively.

\section{Results}

The observations have been carried out in the I band only, due to the faintness of the target stars in bluer filters. Concerning Asiago data, the photometric data of five sufficiently bright ( $0.5-1.5 \mathrm{mag}$ brighter than the targets) and stable stars have been combined to obtain an artificial comparison star with a rms scatter of $\sim 2$ mmag (see the left panels of Fig. 2). The time series for the target stars were calculated by adopting differential photometry with respect to the artificial comparison star. As a result, CFHT BD2 does not show significant light variations in the present data. Conversely, CFHT BD3 is more interesting. As shown in the upper-left panel of Fig. 2, the light curve is indicative of periodic variability. The periodogram analysis (see upper-right panel) yields $f \sim 5.0 \pm 2.4 \mathrm{~d}^{-1}$ (period range $3-7 \mathrm{~h}$ ), with an amplitude of $8 \mathrm{mmag}$ and $S / N \sim 5$. For comparison, we report in the lower-left and right panels of Fig. 2 the light curve and DFT of another star present in the field (named star \#24) with a magnitude comparable to that of CFHT BD3. We see that the noise can hardly be responsible for the light variability displayed by CFHT BD3.

As for the Loiano data, the poor length of the time series prevents us from obtaining reliable results. However, a Fourier analysis of the data on CFHT BD3 presents an indication of a marginally significant $(S / N \sim 3.6)$ periodicity on a time scale shorter than the Asiago observations, $\sim 15 \mathrm{~d}^{-1}$ (period $\sim 1.5 \mathrm{~h}$ ). 


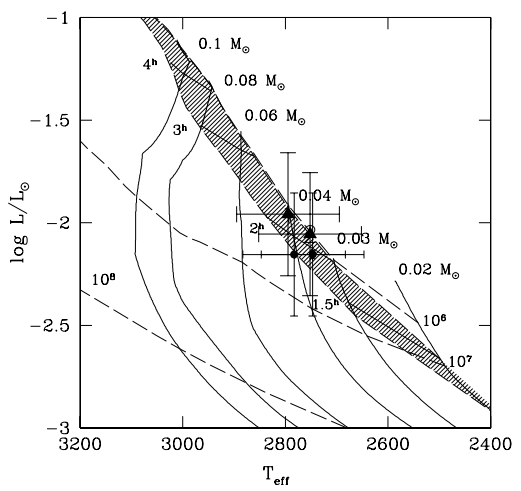

Figure 1: The location of CFHT BD2 and BD3 in the HRD. Triangles: data from Briceño et al. (2002). Circles: data from Grosso et al. (2007). Shaded region: predicted D-burning instability strip of PB05. Evolutionary tracks and isochrones are from Baraffe et al. (1998). Isoperiod curves (in hr) are also shown.
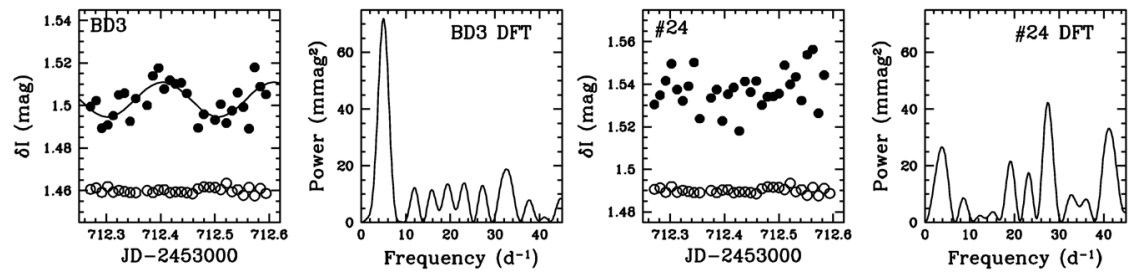

Figure 2: From left, panel 1: Full and empty dots show the Asiago data for CHFT BD3 and for the artificial comparison star, respectively; the solid line is a fit to the data; panel 2: DFT for BD3; panels 3 and 4: as panels 1 and 2, respectively, but for star \#24.

\section{Conclusions}

These preliminary data indicate that CFHT BD3 in Tau-Aur might be a periodic variable with a period in the range $\sim 3-7 \mathrm{~h}$. This interval is larger than the one expected from pulsation models by PB05, given the BD location in the HR diagram (i.e. $\sim 2 \mathrm{~h}$, see Fig. 1). Considering the large uncertainty of the period determination, we must wait for more extended time series observations for a firm conclusion. In any case, only the detection of persistent periodic, or possibly multi-periodic, light variations with time scale of few hours in CFHT BD3 and similar candidates can offer the evidence that the observed variations can be ascribed to pulsation instead of other mechanisms (such as BD rotation, spot modulation) that have been invoked to explain fast variability observed in some BDs (e.g. Caballero et al. 2004).

Acknowledgments. We thank the Asiago and Loiano Observatories for their help with the observations. Partial financial support was provided by PRIN-INAF 2005 under the project "Stellar clusters as probes of stellar formation and evolution" (P.I. Francesco Palla).

\section{References}

Baraffe I., Chabrier G., Allard F., Hauschildt P. H., 1998, A\&A, 337, 403

Briceño C., Luhman K. L., Hartmann L., Stauffer J. R., Kirkpatrick J. D., 2002, ApJ, 580, 317

Caballero J. A., Béjar V. J. S., Rebolo R., Zapatero Osorio M. R., 2004, A\&A, 424, 857

Grosso N., Briggs K. R., Güdel M., et al., 2007, A\&A, in press

Palla F., Baraffe I., 2005, A\&A, 432, L57 